\title{
Erratum to: Diagnosis implications of the whole genome sequencing in a large Lebanese family with hyaline fibromatosis syndrome
}

Zahraa Haidar', Ramzi Temanni², Eliane Chouery ${ }^{1}$, Puthen Jithesh², Wei Liu ${ }^{3}$, Rashid Al-Ali², Ena Wang ${ }^{3}$, Francesco M Marincola ${ }^{4}$, Nadine Jalkh', Soha Haddad ${ }^{5}$, Wassim Haidar ${ }^{6}$, Lotfi Chouchane ${ }^{7}$ and André Mégarbané ${ }^{8^{*}}$

\section{Erratum}

Shortly after the publication of this article [1], one of the authors noticed that his name had been misspelled. The article has been updated. 'Puthen Jitesh' has been changed to 'Puthen Jithesh'.

\footnotetext{
Author details

${ }^{1}$ Unité de Génétique Médicale, Faculté de Médecine, Université Saint-Joseph, Beirut, Lebanon. ${ }^{2}$ Bioinformatics Division, Sidra Medical \& Research Center, Doha, Qatar. ${ }^{3}$ Genomics Core Laboratory, Translational Medicine Division, Sidra Medical \& Research Center, Doha, Qatar. ${ }^{4}$ Research office, Sidra Medical \& Research Center, Doha, Qatar. ${ }^{5}$ Department of Radiology, Hotel Dieu de France University hospital-Beirut, Beirut, Lebanon. ${ }^{6}$ Department of General surgery, Dar Al Amal University Hospital-Baalbeck, Baalbeck, Lebanon. ${ }^{7}$ Laboratory of Genetic Medicine and Immunology, Weill Cornell Medicine-Qatar, Doha, Qatar. ${ }^{8}$ Institut Jérôme Lejeune, 37, rue des Volontaires, Paris 75015, France.
}

Received: 24 January 2017 Accepted: 27 January 2017

Published online: 01 February 2017

\section{References}

1. Zahraa H, Ramzi T, Eliane C, Puthen J, Wei L, Rashid A-A, Ena W, Marincola FM, Nadine J, Soha H, Wassim H, Lotfi C, André M. Diagnosis implications of the whole genome sequencing in a large Lebanese family with hyaline fibromatosis syndrome. BMC Genet. 2017;18:3. doi:10.1186/s12863-017-0471-0. 